\title{
On extension of near-wall domain decomposition to turbulent compressible flows
}

DOI:

10.1016/j.compfluid.2020.104629

\section{Document Version}

Accepted author manuscript

Link to publication record in Manchester Research Explorer

\section{Citation for published version (APA):}

Petrov, M., Utyuzhnikov, S., Chikitkin, A., \& Titarev, V. (2020). On extension of near-wall domain decomposition to turbulent compressible flows. Computers \& Fluids, 210. https://doi.org/10.1016/j.compfluid.2020.104629

\section{Published in:}

Computers \& Fluids

\section{Citing this paper}

Please note that where the full-text provided on Manchester Research Explorer is the Author Accepted Manuscript or Proof version this may differ from the final Published version. If citing, it is advised that you check and use the publisher's definitive version.

\section{General rights}

Copyright and moral rights for the publications made accessible in the Research Explorer are retained by the authors and/or other copyright owners and it is a condition of accessing publications that users recognise and abide by the legal requirements associated with these rights.

\section{Takedown policy}

If you believe that this document breaches copyright please refer to the University of Manchester's Takedown Procedures [http://man.ac.uk/04Y6Bo] or contact uml.scholarlycommunications@manchester.ac.uk providing relevant details, so we can investigate your claim.

\section{OPEN ACCESS}




\title{
On Extension of
}

\section{Near-wall Domain Decomposition to}

\section{Turbulent Compressible Flows}

\author{
Petrov, M. ${ }^{1}$, Utyuzhnikov, S. ${ }^{1,2,3}$, Chikitkin, A. ${ }^{1}$ and Titarev, V. ${ }^{1,4}$ \\ ${ }^{1}$ Moscow Institute of Physics \& Technology, Dolgoprudny 141700, Russia \\ 2 School of Mechanical, Aerospace \& Civil Engineering, \\ the University of Manchester, Manchester, M13 9PL, U.K., \\ e-mail: s.utyuzhnikov@manchester.ac.uk \\ ${ }^{3}$ Skolkovo Institute of Science and Technology, Nobel St., 3, \\ 143025, Moscow, Russia \\ ${ }^{4}$ Federal Research Center "Computer Science and Control" of RAS, \\ 44-2 Vavilov Str., 119333, Moscow, Russia
}

\begin{abstract}
For modeling turbulent flow, the near-wall domain decomposition (NDD) approach initially proposed by the second author and recently developed in a number of papers proved to be very efficient. It leads to a non-overlapping domain decomposition with a Robin-to-Dirichlet map between an inner (near-wall) and outer regions. The regions are linked with each other via interface boundary conditions of Robin type which equivalently replace both the boundary conditions at the wall and simplified governing equations in the inner region. As has been shown, this approach can reduce the computational time by one order of magnitude while retaining sufficiently high accuracy. In the current paper, for the first time the technique is extended
\end{abstract}


to compressible gas flows. In addition, it is modified to include an exact domain decomposition applied to the original Reynolds-averaged Navier-Stokes equations (RANS) without any simplifications near the wall. The efficiency and accuracy of the algorithm are demonstrated on a number of test cases with the use of the SpalartAllmaras turbulence model for compressible flows implemented in the in-house code "FlowModellium". Apart from the approximate NDD (ANDD) based on the thin boundary layer model, for the first time an exact NDD (ENDD) is implemented. The interface boundary conditions in both ANDD and ENDD approaches are consistent. Thereby, the ENDD can effectively complete the ANDD approach when it is needed.

Key words: Near-wall domain decomposition; interface boundary condition; wall function; turbulence; low-Reynolds-number model; steady problems; RANS; compressible flows.

\section{Introduction}

In the modern computational fluid dynamics, adequate prediction of turbulent boundary layers remains one of the main challenges. Due to no-slip boundary conditions and damping effect, a very thin laminar sublayer is formed near the wall. Despite the thickness of this area is only about $1 \%$ of the typical space scale in the normal to the wall direction, its resolution takes up to $90 \%$ of the total computational time [1]. One can expect that for engineering applications domain decomposition should be an effective approach to tackle this problem. The simplest way to implement a domain decomposition is based on wall functions (WFs) [3], [4]. For that the Reynolds Averaged Navier-Stokes

Email address: s.utyuzhnikov@manchester.ac.uk (Petrov, M. ${ }^{1}$, Utyuzhnikov, S. ${ }^{1,2,3}$, Chikitkin, A. ${ }^{1}$ and Titarev, V. ${ }^{1,4}$ ). 
(RANS) equations are considered in an isotropic formulation. Then, the effect of the wall is taken into account via the use of semi-empirical off-wall Dirichlet boundary conditions typically formulated at the centre of cells adjacent to the wall.

Although WFs are widely used in engineering applications because of their computational efficiency, their application range is very limited. In particular, they often contain tunable parameters and do not take into account the effects of pressure gradient and other forces. The computed solution can be mesh sensitive and applications of WFs to flows with complex geometries and boundary layer separation are very problematic. There exist advanced WFs (see e.g. [5], [6], [7], [8]) in which some of the problems mentioned above are resolved. However, they are not able to overcome all the problems since they have a fairly limited basis.

The near-wall domain decomposition (NDD) represents an alternative to the WFs. It was proposed in [11], [12] and [13] as an algorithm to transfer the boundary condition from the wall to an interface boundary. If in the inner region the governing equations correspond to a thin boundary layer (TBL) model, then the interface boundary conditions (IBCs) are always of Robin type. They can equivalently replace the governing equations in the inner region [14]. As soon as the solution in the outer region is obtained, the innerregion solution can immediately be calculated. Thus, the NDD amounts to a non-overlapping domain decomposition. The IBCs are mostly formulated in a uniform manner for all dependent variables. They are mesh independent and do not contain free parameters. Moreover, IBCs of Robin type are more robust than those of Dirichlet or Neumann type [15]. As shown in [16] and [17], in the case of multidimensional and unsteady models in the inner region, the IBC 
must be nonlocal in space and time, respectively. In [16] nonlocal boundary conditions are implemented via an approximation of the Steklov-Poincaré operator. The unsteady IBC contain a memory term, and its presence can play a crucial role for the accuracy of prediction in the case of essentially unsteady flows as has recently been shown in $[24,26]$ for a pulsating channel laminar flow.

The NDD approach outlined above is approximate since the resolution of the inner region is based on a thin-layer model. In addition, it assumes an approximate profile for the turbulent viscosity coefficient in the inner region to be known. Thus, there is a trade-off between the accuracy and computational time which is determined by the location of the interface boundary. If it is situated closer to the wall, then the accuracy and computational cost both increase and vice versa. The efficiency of NDD for engineering applications was demonstrated in [18], [22] and [23]. It was shown that the NDD allows the computational time to be reduced as much as one order of magnitude while retaining sufficiently high accuracy.

So far the NDD has not been used for modeling compressible gas flows. In addition, its application becomes very problematic for complex geometries and transition regimes. Although the NDD was successfully used to laminarturbulent transition in the framework of the boundary layer model [2], its use for the Navier-Stokes equations failed because of a poor approximation for the turbulent viscosity coefficient in the inner region.

In the present paper, the domain decomposition algorithm is extended to compressible gas. The extension requires a resolution of the density in the inner region. A principal finding is related to the continuity equation. This equation 
is not taken into account by the main algorithm of NDD. As noted in [18], the conservation law of mass can be violated. To prevent this, an integral algorithm was proposed which was based on the assumption of incompressible fluid. In the present paper an alternative is developed. In addition, to make the entire algorithm more universal, the approximate NDD approach is modified to an exact NDD in which full RANS equations are solved in the inner region. The resulting new method is implemented in the in-house code "FlowModellium" $[19,20,21]$, which is used to run all presented calculations.

The rest of the paper is organized as follows. Section 2 contains the governing equations. The basic numerical method is formulated in Section 3. In Section 4 formulation and implementation of approximate near-wall decomposition (ANDD) are discussed. Exact near-wall decomposition (ENDD) is described in Section 5. Section 6 contains computational tests for ANDD and ENDD methods. The compressible flow over the following geometries are considered: plane with cylindrically blunted leading edge, half cylinder, compression corner and a body with sharp edge. Comparison of ANDD and ENDD approaches with respect to accuracy and computational cost is provided for the half cylinder flow. All computed results are compared with the one-block solution (without decomposition). Analysis of such comparisons is provided. Finally, conclusions are drawn in Section 7.

\section{Governing equations and turbulence model}

Gas state at position $\boldsymbol{x}=\left(x_{1}, x_{2}, x_{3}\right)^{T}$ at time moment $t$ is characterized by density $\rho$, velocity $\boldsymbol{u}=\left(u_{1}, u_{2}, u_{3}\right)^{T}$, pressure $p$ and energy per unit volume 
$E$. In the dimensional form the governing equations are given by

$$
\frac{\partial}{\partial t} \boldsymbol{U}+\nabla\left(\boldsymbol{F}-\boldsymbol{F}^{v}\right)=\boldsymbol{S}(\boldsymbol{U}), \quad \boldsymbol{F}=\left(\boldsymbol{F}_{1}, \boldsymbol{F}_{2}, \boldsymbol{F}_{3}\right), \quad \boldsymbol{F}^{v}=\left(\boldsymbol{F}_{1}^{v}, \boldsymbol{F}_{2}^{v}, \boldsymbol{F}_{3}^{v}\right),
$$

where $\boldsymbol{U}$ is vector of conserved variables, $\boldsymbol{F}, \boldsymbol{F}^{v}$ are tensors of convective and viscous fluxes, $\boldsymbol{S}$ is the source term:

$$
\boldsymbol{U}=\left(\begin{array}{c}
\rho \\
\rho u_{1} \\
\rho u_{2} \\
\rho u_{3} \\
E
\end{array}\right), \quad \boldsymbol{F}_{k}=\left(\begin{array}{c}
\rho u_{k} \\
\rho u_{1} u_{k}+\delta_{1 k} p \\
\rho u_{2} u_{k}+\delta_{2 k} p \\
\rho u_{3} u_{k}+\delta_{3 k} p \\
(E+p) u_{k}
\end{array}\right), \quad \boldsymbol{F}_{k}^{v}=\left(\begin{array}{c}
0 \\
\tau_{1 k} \\
\tau_{2 k} \\
\tau_{3 k} \\
u_{\alpha} \tau_{\alpha k}-q_{k}
\end{array}\right)
$$

Here, $\tau_{i j}, q_{k}$ are components of stress tensor and heat flux vector, $p=\rho R_{g} T\left(R_{g}\right.$ is gas constant), $E=\rho\left(e+\frac{1}{2} u_{\alpha} u_{\alpha}\right)$ ( $e$ is internal energy per unit mass). For the laminar flow the source term is $\boldsymbol{S}=\mathbf{0}$. For turbulent flows a modification SAnonft2 of Spalart-Allmaras model is used [25]. Equations (1) are supplemented by an additional equation, so that vectors $\boldsymbol{U}, \boldsymbol{F}_{k}, \boldsymbol{F}_{k}^{v}, \boldsymbol{S}$ have an additional 6th component

$$
U(6)=\rho \tilde{\nu}, F_{k}(6)=\rho \tilde{\nu} u_{k}, \quad F_{k}^{v}(6)=\frac{\rho}{\sigma}\left((\nu+\tilde{\nu}) \frac{\partial \tilde{\nu}}{\partial x_{k}}\right), \quad S(6)=S_{s a} .
$$

Here, $\tilde{\nu}$ is the turbulent variable, the full viscosity is given by $\mu=\mu_{m o l}+\mu_{t}$, full heat conductivity $\lambda=\lambda_{m o l}+\lambda_{t}$, where $\mu_{m o l}$ is molecular viscosity, $\mu_{t}$ turbulent viscosity

$$
\mu_{t}=\rho \tilde{\nu} f_{v 1}
$$

$\lambda_{m o l}$ is the molecular heat conductivity coefficient, $\lambda_{t}$ is the turbulent heat 
conductivity. Then, we have

$$
\begin{gathered}
S_{s a}=c_{b 1} \rho \tilde{S} \tilde{\nu}+c_{w 1} f_{w} \rho\left(\frac{\tilde{\nu}}{d}\right)^{2}+c_{b 2} \frac{\rho}{\sigma} \frac{\partial \tilde{\nu}}{\partial x_{k}} \frac{\partial \tilde{\nu}}{\partial x_{k}} \\
\tilde{S}=S+\frac{\tilde{\nu}}{\kappa^{2} d^{2}} f_{v 2}, \quad S=\sqrt{2 W_{i j} W_{i j}}, \quad W_{i j}=\frac{1}{2}\left(\frac{\partial u_{i}}{\partial x_{j}}-\frac{\partial u_{j}}{\partial x_{i}}\right) \\
f_{v 1}=\frac{\chi^{3}}{\chi^{3}+c_{v 1}^{3}}, \quad \chi=\frac{\tilde{\nu}}{\nu_{m o l}}, \quad f_{v 2}=1-\frac{\chi}{1+\chi f_{v 1}}, \quad f_{w}=g\left[\frac{1+c_{w 3}^{6}}{g^{6}+c_{w 3}^{6}}\right]^{1 / 6} \\
g=r+c_{w 2}\left(r^{6}-r\right), r=\min \left(\frac{\tilde{\nu}}{\tilde{S} \kappa^{2} d^{2}}, 10\right),
\end{gathered}
$$

where the values of the coefficients are

$$
\begin{aligned}
& c_{b 1}=0.1355, c_{b 2}=0.622, \quad c_{v 1}=7.1, \\
& c_{w 2}=0.3, \quad c_{w 3}=2, \quad c_{w 1}=\frac{c_{b 1}}{k^{2}}+\frac{1+c_{b 1}}{\sigma}, \\
& \sigma=2 / 3, \quad \kappa=0.41 .
\end{aligned}
$$

The turbulent heat conduction coefficient is given by $\lambda_{t}=c_{p} \mu_{t} / \operatorname{Pr}_{t}$; with the turbulent Prandtl number $\operatorname{Pr}_{t}=0.85$.

While setting up boundary conditions, the turbulent viscosity at the wall is set to zero, whereas at the free-stream it is assumed to be triple value of the molecular free-stream viscosity.

\section{$3 \quad$ Numerical method}

The outlined equations are solved using the implicit finite-volume numerical method, described in $[19,20,21]$ and implemented in the in-house code "FlowModellium". Let us re-write system (1) by integrating it in each spatial 
cell $C_{i}$ in the computational domain and using Gauss-Ostrogradsky theorem as follows:

$$
\left(\bar{U}_{i}\right)_{t}=R_{i}=-\frac{1}{\left|C_{i}\right|} \sum_{f_{j} \in \mathcal{F}\left(C_{i}\right)}\left(\Phi_{j i}-\Phi_{j i}^{v}\right)+\bar{S}_{i}, \quad i=1, \ldots, N_{c}
$$

where $\bar{U}_{i}$ is the cell average of the unknown vector. Inviscid fluxes $\Phi_{j i}$ are approximated using the Riemann problem solution, viscous fluxes $\Phi_{j i}^{v}$ and the source term $\bar{S}_{i}$ are computed by using the approximation of the involved spatial derivatives. As a result, system (4) becomes the system of nonlinear ODEs:

$$
\frac{d W(t)}{d t}=R(W(t))
$$

where

$$
W=\left[\begin{array}{c}
\bar{U}_{1} \\
\bar{U}_{2} \\
\cdots \\
\bar{U}_{N_{c}}
\end{array}\right], \quad R(W)=\left[\begin{array}{c}
R_{1}(W) \\
R_{2}(W) \\
\ldots \\
R_{N_{c}}(W)
\end{array}\right]
$$

The use of an implicit Euler approximation in time results in the following algebraic system of equations for time increments $\Delta W$

$$
\left[I-\Delta t \frac{\partial R}{\partial W}\left(W^{n}\right)\right] \Delta W=A \Delta W=\Delta t R\left(W^{n}\right)
$$

which is solved using LU-SGS scheme [27,28]. Its modifications in the context of the present method are discussed in $[21,20]$. The stationary solution is found by time marching until the following condition is satisfied

$$
\left\|R\left(W^{n}\right)\right\| /\left\|R\left(W^{1}\right)\right\|<\epsilon
$$


where usually we use $\epsilon=10^{-6}$.

For efficient solution of the problem the current work employs the near-wall domain decomposition method, outlined in detail below. In order to implement the method, the computational domain is split into two parts: inner near-wall part and external part. Two approaches are considered in the present work: ANDD and ENDD approaches. In both ENDD and ANDD the boundary conditions of Robin type at the interface boundary are formally consistent. Moreover, in both approaches the governing equations are solved in the outer region without any simplifications. However, in the ANDD the governing equations are simplified in the inner region using the thin-layer (TBL) model whereas in ENDD full governing equations are solved.

It should be noted that high-Re turbulence models use rather coarse computational meshes with the larger first cell size. One of the advantages of ANDD is that there is no need to construct a separate mesh. The near-wall inner region can be constructed directly using a low-Re computational mesh. Such a mesh can be immediately used in the outer region. In the inner region the mesh can be locally generated from each node at the interface boundary to the wall. In the next section the implementation of ANDD is outlined.

\section{Approximate near-wall domain decomposition}

As noted above, in the case of ANDD in the inner region the Navier-Stokes equations are simplified to the TBL model, in which only the terms contributing to the boundary layer model are retained. First, we demonstrate the main idea of the ANDD in application to a TBL equation defined in $0 \leq y \leq y_{e}$, 
where $y$ is the coordinate towards the normal to the wall:

$$
\frac{\partial}{\partial y}\left(\mu \frac{\partial U}{\partial y}\right)=f, \quad U(0)=U_{0}, \quad U\left(y_{e}\right)=U_{1}
$$

Here, $U$ is a dependent variable, $f$ is some right-hand side.

Next, we introduce a near-wall inner region $0 \leq y \leq y^{*}$ and outer region $y^{*} \leq y \leq y_{e}$. It is turned out one can exactly transfer the boundary condition from the wall $(y=0)$ to interface boundary $y=y^{*}[18]$ :

$$
U^{*}=\left.\frac{\partial U}{\partial y}\right|_{y^{*}} f_{1}+f_{2}+U_{0}
$$

where

$$
f_{1}=\int_{0}^{y^{*}} \frac{\mu^{*}}{\mu} d y, \quad f_{2}=-\int_{0}^{y^{*}} \frac{1}{\mu} \int_{0}^{y^{\prime}} f d y d y^{\prime}, \quad \mu^{*}=\mu\left(y^{*}\right) .
$$

In this way, the IBC can be formulated in a universal form (9) for all primitive variables except the normal velocity.

It should be noted that under some conditions unsteady terms have to be taken into account in the derivation of IBC [29]. In this case, the IBC contains a memory term. A detailed analysis of such regimes as well as the derivation of unsteady IBC is provided in [29] and beyond the scope of the current paper.

The general framework for solving the problem by the approximate near-wall decomposition method can be formulated as follows:

(1) specify the initial flow field in both inner and outer domains;

(2) in the inner region the TBL model is used to obtain the boundary condition of Robin type at the interface boundary;

(3) the problem is solved in the outer region using Robin boundary condition 
(9) at the interface boundary. This step corresponds to one global LUSGS iteration of Eq. (7) in the outer region;

(4) once the solution in the outer region is found, the flow field in the inner region is recalculated with Dirichlet boundary conditions obtained from the solution in the outer region;

(5) if the convergence condition (8) is not satisfied, return to step 2.

For the numerical method, outlined in step 3, the formulation of the boundary condition at the interface boundary boils down to specifying the both inviscid $\Phi_{j i}$ and viscous $\Phi_{j i}^{v}$ numerical fluxes in Eq. (4). To compute this flux, we need to know the values of all primitive variables at the boundary, which are found from the corresponding conservation laws complete with the boundary condition of Robin type.

Let us write down the calculation procedure for both the boundary conditions at the interface and the solution in the inner region after the solution in the outer region is found.

\subsection{Computation of the tangential velocity}

Using the thin-layer model, the conservation of momentum in the inner region can be written in the following form:

$$
\frac{\partial}{\partial y}\left(\mu \frac{\partial v_{\tau}}{\partial y}\right)=\frac{d p}{d x}
$$

Here, $v_{\tau}$ is the value of the tangential velocity. Integrating Eq. (10) twice, the following condition of Robin type is obtained at the interface boundary [18]:

$$
v_{\tau}^{*}=f_{1}^{v} v_{\tau, y}^{*}+f_{2}^{v},
$$


which can be approximated as

$$
v_{\tau}^{*}=\frac{v_{\tau, I} f_{1}^{v}+d f_{2}^{v}}{d+f_{1}^{v}}
$$

Here, $v_{\tau}^{*}$ and $v_{\tau, I}$ are the values of the tangential velocity at the interface boundary and in in the first cell near the interface in the outer region; $f_{1}^{v}$ and $f_{2}^{v}$ values of integrals for the tangential component of velocity; $d$ distance from the center of the first cell to the interface boundary.

The profile of the tangential velocity in the inner domain can be obtained from the momentum conservation law, simplified as in the TBL model, with closure (11):

$$
v_{\tau}(y)=\left.\frac{d v}{d y}\right|_{y^{*}} \int_{0}^{y} \frac{\mu^{*}}{\mu(\xi)} d \xi-\int_{0}^{y} \frac{\frac{d p}{d x}\left(y^{*}-\xi\right)}{\mu(\xi)} d \xi
$$

\subsection{Computation of the normal component of velocity}

As was noted in [22], interface boundary condition (11) does not take into account continuity equation. Therefore, it does not guarantee mass conservation. In [22] for the near-wall decomposition of the incompressible gas a Dirichlet-type boundary condition for the normal component $v_{n}$ of velocity was put forward

$$
v_{n}\left(y^{*}\right)=K \int_{0}^{y^{*}} \frac{\xi^{4} d \xi}{\mu+\mu_{t}(\xi)}
$$

where $K$ is computed from the value in the first cell near the interface boundary. In order to satisfy the continuity equation in the complete domain, this condition has to be corrected. To do so, the mass flux $\dot{m}_{S}$ through each mesh face at the interface boundary has to be computed, and the value of $v_{n}$ is 
corrected as follows:

$$
v_{n}\left(y^{*}\right)=K \int_{0}^{y^{*}} \frac{\xi^{4} d \xi}{\mu+\mu_{t}(\xi)}-\frac{\dot{m}_{S}}{\rho S}
$$

In the present paper, an interface condition of Robin type for the normal component of the velocity which takes into account the continuity condition is derived. In this case there is no need to carry out an additional correction for the mass flux. Using Taylor expansion and taking into account impermeability condition and continuity equation, the normal velocity can be expressed as follows (terms higher than 2nd order are dropped):

$$
v_{n} \approx \alpha \frac{y^{2}}{2}+\beta \frac{y^{3}}{6}
$$

From the motion equations it follows that $\alpha=\frac{p_{y}}{\mu}$, where $p_{y}$ is pressure derivative with respect to $y, \mu$ is the laminar viscosity at the wall. Then

$$
v_{n}^{\prime}=\alpha y+\beta \frac{y^{2}}{2} .
$$

Eliminating $\beta$, a condition at the interface boundary $y^{*}$ is obtained:

$$
v_{n}=\frac{y^{*}}{3} v_{n}^{\prime}+\frac{y^{* 2}}{6} \frac{p_{y}}{\mu} .
$$

Finally, after dropping 2nd-order terms, the Robin condition at the interface boundary for the normal component of velocity takes the following form:

$$
v_{n}=\frac{y^{*}}{3} v_{n}^{\prime}
$$

After approximating Eq. (13) near interface boundary, the normal component of velocity near $y^{*}$ can be computed as

$$
v_{n}=\frac{y^{*}}{3 d+y^{*}} v_{n, I}
$$


where $v_{n, I}$ is the value of the normal component of velocity in the first cell near interface in the outer region.

Once the solution in the outer region is computed, the profile of the normal component of velocity can be obtained using formula:

$$
v_{n}(y)=v_{n}\left(y^{*}\right)\left(\frac{y}{y^{*}}\right)^{2} .
$$

4.3 Temperature interface condition and computation of the density profile in the inner region

Simplified energy equation in the inner region has the following form

$$
\frac{\partial}{\partial y}\left(k \frac{\partial T}{\partial y}\right)=0
$$

Integrating Eq. (14) twice, the condition of Robin type at the interface boundary is obtained:

$$
T^{*}=\frac{d T}{d y}\left(y^{*}\right) f_{1}^{T}+T_{w},
$$

where $f_{1}^{T}$ is the value of the temperature integral; $T^{*}$ temperature value at interface; $T_{w}$ surface temperature. Once the solution in the inner region is found, the temperature is computed from (14) with the closure (15):

$$
T(y)=\left.\frac{d T}{d y}\right|_{y^{*}} \int_{0}^{y} \frac{k^{*}}{k(\xi)} d \xi+T_{w} .
$$

For computation of the density profile in the inner region the assumption of the constant pressure in the normal direction to the wall is used. Density profile $\rho=\rho(y)$ in the inner region is obtained from the pressure value $p_{I}$ in the first cell near the interface and temperature profile $T=T(y)$ inside the 
inner region from the equation of state of ideal gas:

$$
\rho(y)=\frac{p_{I}}{T(y) R} .
$$

The consideration of the compressibility effects allows us to compute profiles of viscosity and friction velocity $u^{*}$ more accurately, which influences computation of integrals $f_{1}$ and $f_{2}$.

4.4 Computation of the turbulent variables. Turbulence profile in the inner region

A viscosity profile in the inner region is needed to compute integrals $f_{1}, f_{2}$ in the boundary conditions of Robin type. In the ANDD method it is assumed that the profile of turbulent viscosity or turbulent variable are known a priori. Therefore, the value of $\tilde{\nu}$ at the interface boundary is readily computed. One of the profiles used here is the one due to Duprat et al. [34]. It takes into account pressure gradient, which allows the separation point to be predicted more accurately:

$$
\begin{gathered}
\mu_{t}(\xi)=\mu \kappa \xi^{*}\left[\alpha+\xi^{*}(1-\alpha)^{3 / 2}\right]^{\beta}\left(1-\exp \left(\frac{-\xi^{*}}{1+A \alpha^{*}}\right)\right)^{2}, \\
\kappa=0.41, \quad A=17, \quad \beta=0.78, \quad \alpha=u_{\tau}^{2} / u_{\tau p}^{2}, \quad \xi^{*}=y u_{\tau p} / \mu \\
u_{\tau p}=\sqrt{u_{\tau}^{2}+u_{p}^{2}}, \quad u_{\tau}=\sqrt{\left|\tau_{w}\right| / \rho}, \quad u_{p}=\left|\frac{\mu}{\rho} \frac{d p}{d x}\right|^{1 / 3} .
\end{gathered}
$$

In case of using the profile from [34], the calculation of the profile of turbulent variable $\tilde{\nu}$ in the inner region requires the use of the following iterative 
procedure with a fixed point based on Eq. (3):

$$
\tilde{\nu}_{i+1}=F\left(\tilde{\nu}_{i}\right)=\frac{\mu}{\rho}\left(\frac{\mu_{t}\left(\frac{\rho^{3} \tilde{\nu}_{i}^{3}}{\mu^{3}}+c_{v 1}\right)}{\rho \nu_{i}}\right)^{1 / 3}
$$

The iterative procedure is initialised with $\tilde{\nu}_{0}=\frac{\mu_{t}}{\rho}$ and practically converges to sufficient accuracy in several iterations. Thus, the operator $F$ is compressible since the convergence occurs for any $y$.

As an alternative, the profile from ZPG SA solution [35] can be used

$$
\tilde{\nu}=\kappa u_{\tau} y
$$

In both cases the surface friction is needed. It can be computed by integrating Eq. (10) in the near-wall region

$$
\tau_{w}=\mu \frac{\partial v_{\tau}}{\partial y}=\frac{\mu^{*}}{f_{1}^{v}}\left(v_{\tau}^{*}-f_{2}^{v}\right)-\frac{d p}{d x} y^{*}
$$

\section{Exact near-wall domain decomposition}

The ENDD can be implemented as an alternative or addition to the ANDD. This method of solution can be used for verification of the ANDD result. ENDD can also be used in the cases when ANDD is not very well suited, e.g. separated flows near very non-smooth surfaces as well as flows with laminarturbulent transition.

Let us consider the equations solved in the exact decomposition. Let $u_{1}$ be the solution of the problem in the inner domain, $u_{2}$ be solution in the outer domain. Then, the solution of the complete problem is reduced to the iterative 
solution of two systems:

$$
L\left(u_{1}\right)=R_{1}, \quad \frac{\partial u_{1}^{k+1}}{\partial n}+\sigma u_{1}^{k+1}=g_{1}^{k+1} \text { at } y=y^{*}
$$

and

$$
L\left(u_{2}\right)=R_{2}, \quad \frac{\partial u_{2}^{k+1}}{\partial n}-\sigma u_{2}^{k+1}=g_{2}^{k+1} \text { at } y=y^{*}
$$

where

$$
g_{1}^{k+1}=g_{2}^{k+1}+2 \sigma u_{2}^{k+1}, \quad g_{2}^{k+1}=g_{1}^{k}-2 \sigma u_{1}^{k}
$$

and

$$
g_{1}^{0}=\frac{\partial u_{1}^{0}}{\partial n}+2 \sigma u_{1}^{0}
$$

It is easy to see that these Robin IBCs are exact. In the general form, they were introduced for the non-overlapping domain decomposition by Lions and Deng [30], [31]. The convergence of iteration process (19)-(21) for an arbitrary positive $\sigma$ in application to the Poisson equation was proven in [32].

The differential operator $L$ in the governing equations is approximated in the same way as in the one-block formulation (without domain decomposition). Both systems are solved with the Robin boundary conditions at the interface boundary. It corresponds to one global LU-SGS iteration of Eq. (7) in each domain.

To make these IBCs consistent with the IBC in ANDD, we select $\sigma=\frac{1}{f_{1}}$, where $f_{1}$ is the integral from the Robin boundary condition (9). This choice of $\sigma$ is based on the locally one-dimensional approximation of the operator Steklov-Poincaré [33]. 


\section{Test cases}

The accuracy and efficiency of both ANDD and ENDD approaches were tested on a number of problems. Although all problems are planar, the calculations were carried out with the use of the in-house 3D code "FlowModellium". Three cells were formally set along the direction orthogonal to the plane with symmetry boundary conditions to convert planar mesh into three-dimensional one.

The ANDD method is tested on the problems of flow past a flat plate with the cylindrically blunted front edge, half cylinder, compression corner and flow past a sharp edge. Additionally for the flow past half cylinder, the ENDD method is used. It should be noted that considered tests include modelling of essentially separated flows and supersonic flows past non-smooth surfaces. Such tests are challenging for the WF-based approaches.

For each test case a low-Re one-block calculation without decomposition is carried out for the comparison purposes. The first cell height in the boundary layer in these calculations is such that $y^{+} \approx 1$. For two-block calculation, the same mesh is used with the explicit division into outer and inner regions. In the two-block solution, the distance from the interface boundary to the wall is $y^{*} \approx 5 \cdot 10^{-4} \mathrm{~m}$. This distance is chosen to make $y^{*+} \approx 100$. It should be noted that using this estimate in the near-wall region the TBL model equations are satisfied with good accuracy. The results are compared by the surface friction distribution.

System (1) is solved using the finite-volume implicit method outlined in Section 3. In the two-block calculation with NDD the conditions of Robin type 
for normal and tangential components of velocity as well as temperature are used at the interface, see Section 4.

\subsection{Flow past a flat plate with the blunted leading edge}

The first test problem for the ANDD method is supersonic flow past a flat plate with a cylindrically blunted leading edge. The free-stream Mach number is $M=1.5, \operatorname{Re}=6 \cdot 10^{6}$ per meter, cylinder radius is $1 \mathrm{~m}$. The position $x=-1$ corresponds to the stagnation point, from $x=0$ the cylindrical part becomes the plate. The free-stream direction is parallel to the plate. There is no separation in this flow so that the main aim of the test is to show that the method can model compressibility effects.

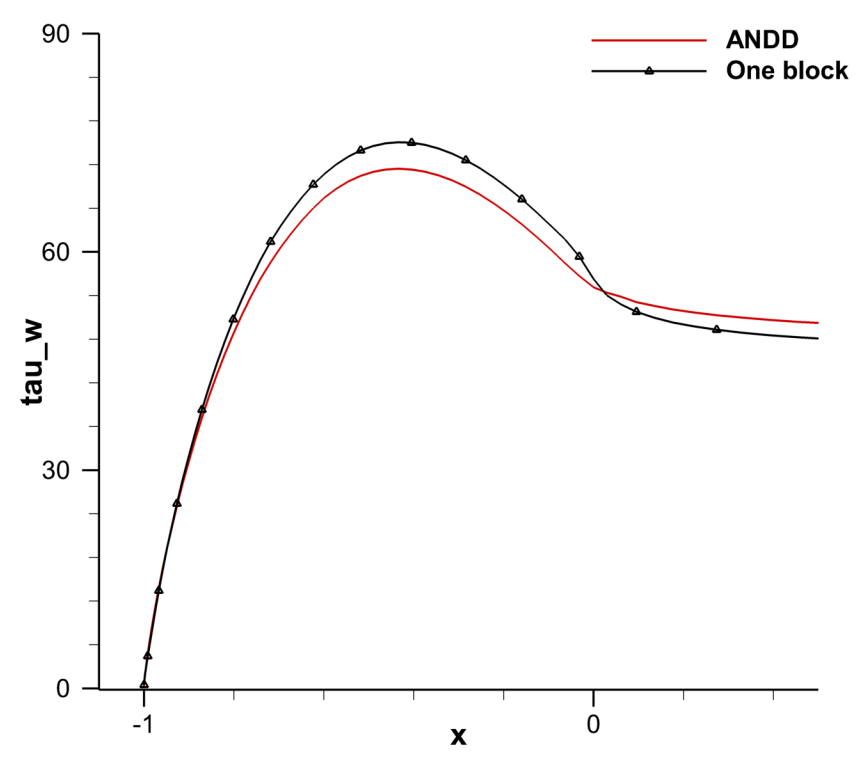

Figure 1. Surface friction on the blunted plate obtained in the one-block calculation and using ANDD

For the one-block calculation the height of the first cell in the boundary layer is $10^{-6} \mathrm{~m}$. In the two-block the distance from the interface boundary to the 
wall corresponds to $y^{*+}=10 \div 140$. Fig. 1 depicts the surface friction on the blunted cylinder using both one-block and ANDD calculations. Good agreement around stagnation point is observed, that is in the area where the compressibility effects are most pronounced. A slight difference can be seen in the region of flow acceleration further down along the cylinder. This region corresponds to the interface position $y^{*+}>100$, where the thin-layer model can give a more pronounced error.

Fig. 2 shows density profile near stagnation point. In the two-block calculation (ANDD), the profile includes both outer and inner (near-wall) computational domains. The presented profiles demonstrate good agreement in the part of the flow domain where compressibility is most pronounced.

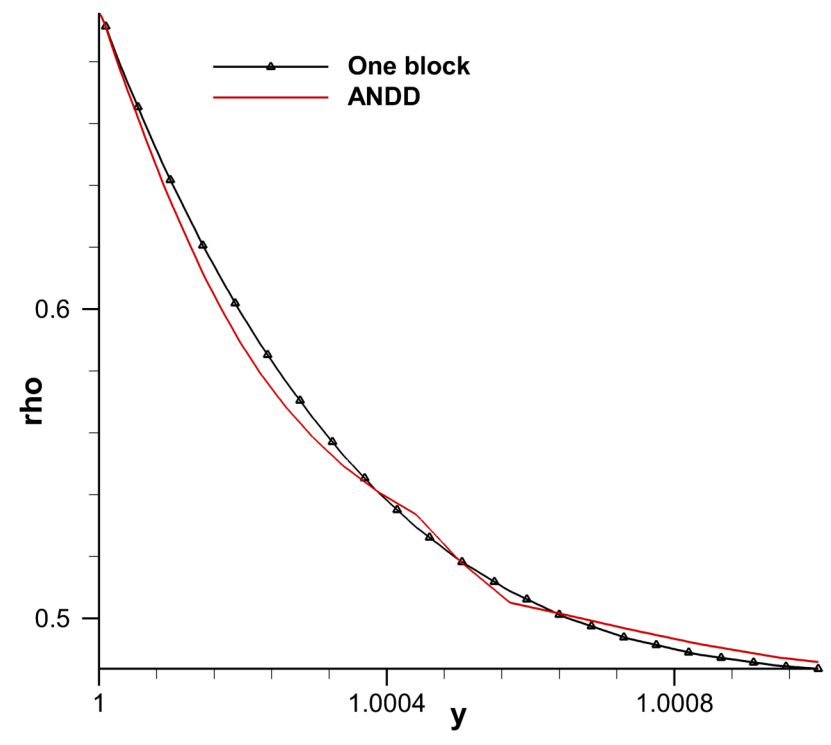

Figure 2. Density profile near stagnation point for the flow over the blunted plate obtained in the one-block calculation (one block) and using ANDD 


\subsection{Flow over half cylinder}

The problem of a stationary flow over half cylinder is a classical problem with flow separation. This test demonstrates capabilities of the approximate and exact decompositions for computing such type of flows. A weakly-compressible flow with $M=0.15$ and $\mathrm{Re}=6 \cdot 10^{6}$ per meter is considered. Cylinder radius is $1 \mathrm{~m}$. The position $x=-1$ corresponds to the front stagnation point, $x=1$, downwind stagnation point. The separation point position in the one-block calculation corresponds to $x \approx 0.42$. The height of the first cell in the boundary layer is $10^{-6} \mathrm{~m}$. For the two-block run the distance from the interface boundary to the wall corresponds to $y^{*+}=10 \div 190$.

Fig. 3 shows comparison of the skin friction between one-block, ANDD and ENDD calculations. It is seen that, as in the previous test, the approximate decomposition demonstrates large discrepancy in the region with higher $y^{+}$ values. It can also be seen that the separation point position for the approximate decomposition is less clear. All these differences are eliminated by the use of the exact (full) decomposition, which is achieved by using the solution of the full governing equations in the inner region. It should be mentioned that the computing time for the exact decomposition is higher than that for the approximate one.

Fig. 4 shows comparison of the residual convergence history (decay) as a function of the computational cost. The computational cost was estimated as follows. There are five times more cells in the outer region than in the inner one. Then, the computation cost of one iteration in the inner region is taken as one unit, in the outer one, five units, of the complete one-block iteration, six 


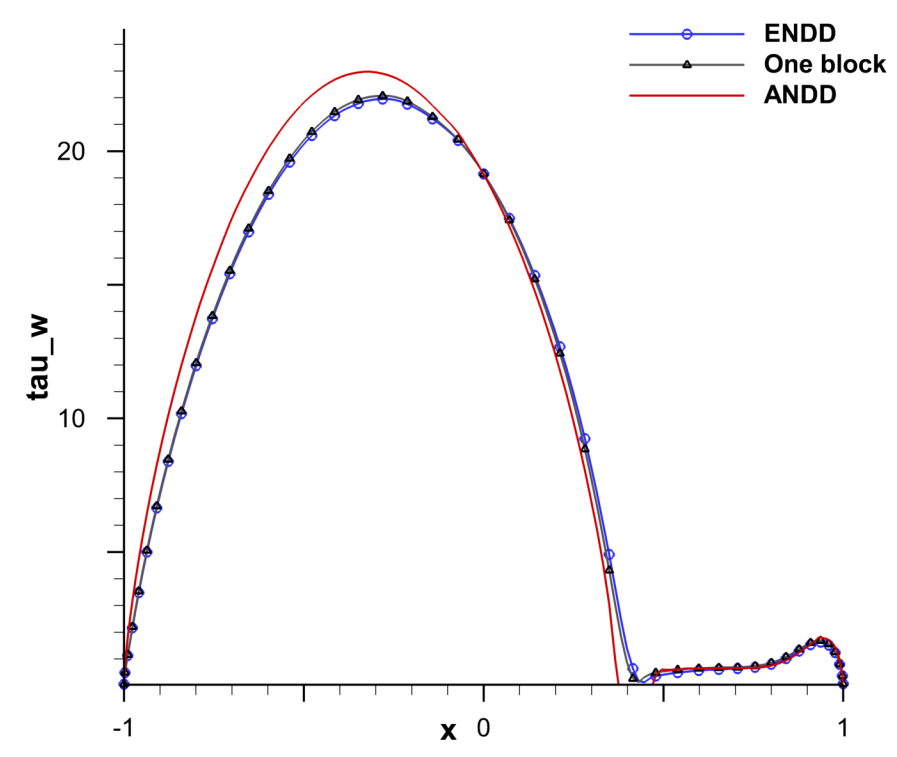

Figure 3. Skin friction on the cylinder computed by using one-block, ANDD and ENDD approaches

units. From the comparison it is seen that two-block ANDD method converges almost three times faster than one-block and ENDD calculations. ENDD and one-block runs converge approximately with the same time. It should also be noted that for all calculations there is a moment when the convergence slows down. However, for the ANDD method it happens much later.

\subsection{Flow past an airfoil with a sharp trailing edge}

This test problem considers flow past a flat airfoil with a circular leading edge and a sharp trailing edge with $25^{\circ}$ angle [9]. Free-stream Mach number is $M=0.15$, Reynolds number computed by the profile length is $\operatorname{Re}=2.15 \times 10^{6}$. The height of the first cell in the boundary layer is $10^{-6} \mathrm{~m}$, which corresponds to $y^{+} \approx 1.5$. For the two-block run the distance from the interface boundary to the wall corresponds to $y^{*+}=100$. Fragment of the computational domain 


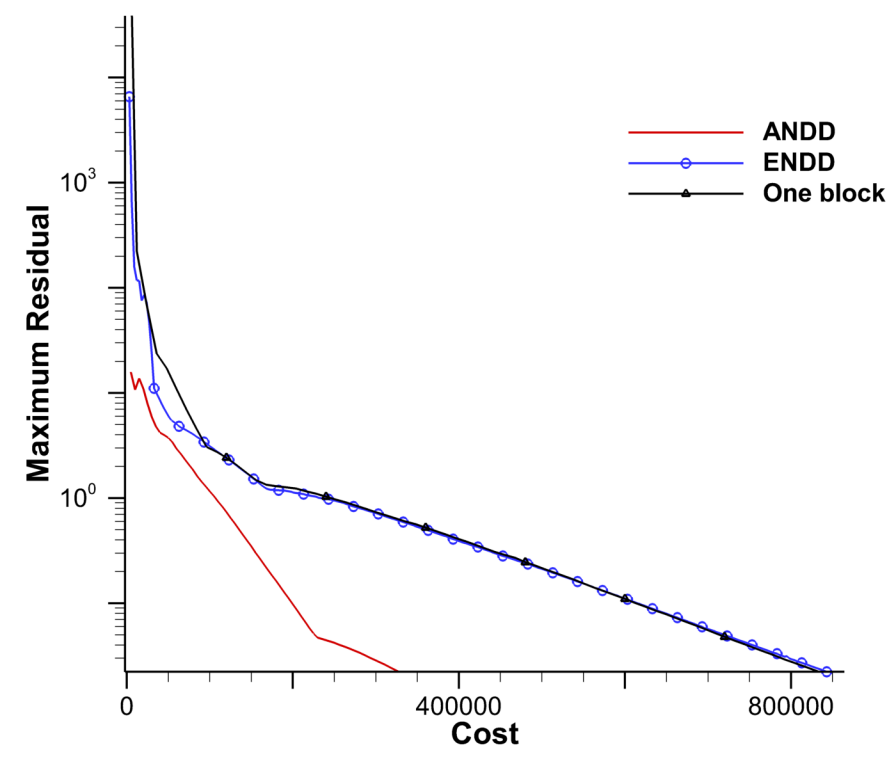

Figure 4. Comparison of the residual time history for ANDD, ENDD and one-block calculations

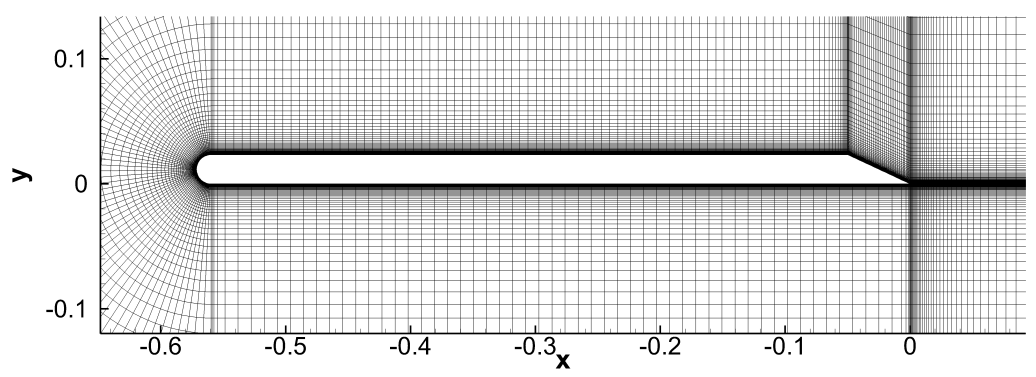

Figure 5. Fragment of the computational domain with mesh for flow past airfoil with trailing edge

with mesh is shown in Fig. 5.

In [9] only the flow near the trailing edge of the profile is considered whereas in the inflow boundary the authors specify the velocity profile of the developed turbulent boundary layer. This is done mainly in order to reduce the computational cost. In the present work the problem is solved in the complete flow domain from the beginning. It is worth noting that in [9] a smoothed 
profile geometry without sharp corners is used whereas in the present work the profile with corners is used in accordance with the original experiment [36]. The presence of sharp kinks in the geometry makes it possible to test the sensitivity of the interface boundary conditions to their presence.

The general flow pattern near the sharp edge is shown on Fig. 6 for both oneblock and two-block calculations. A separation zone is formed after the obtuse angle of the profile, good resolution of which requires correct prediction of all flow characteristics.

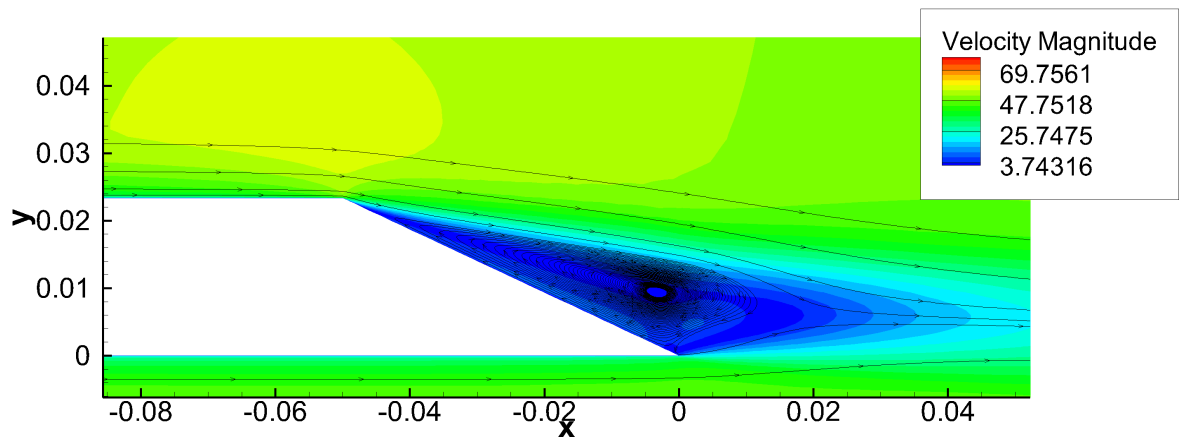

(a) One-block solution

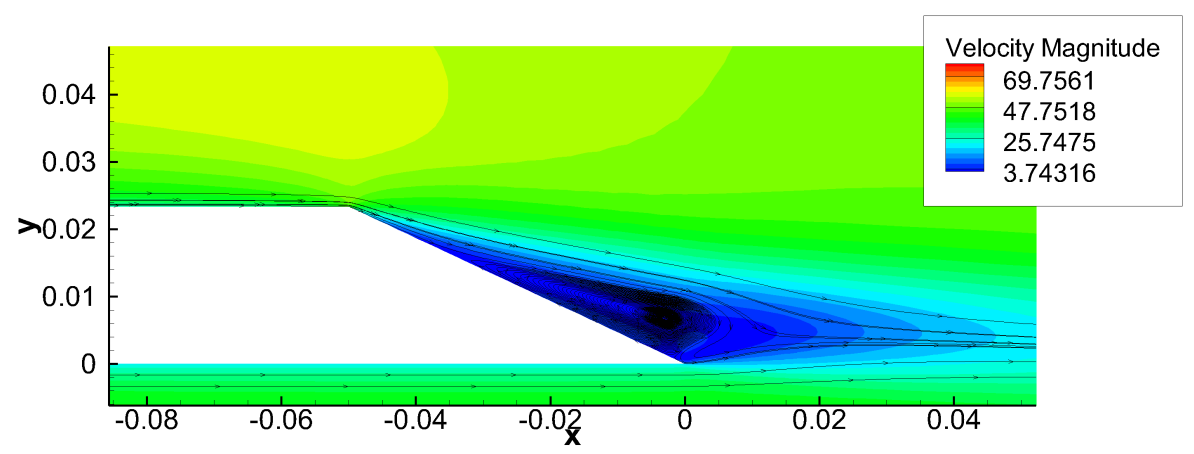

(b) Two-block solution

Figure 6 . Velocity magnitude contour levels near the sharp edge of the profile

Fig. 7 shows dependence of skin friction coefficient on the longitudinal coordinate (values over the lower side are shown negative). Position $x / h=0$ corresponds to the sharp edge. Shown is the comparison with time averaged 
Large Eddy Simulation (LES) values from [9], which were obtained for the smoothed geometry.

The solution is computed using the interface boundary conditions with the viscosity profile approximation [10] which takes into account pressure gradient. It is seen that in the obtained solution the beginning of the separation zone moves close to the sharp edge. This is probably caused by the turbulence viscosity and turbulence variable behaviour: near the kink of the profile behaviour of turbulent variable is very different from the one-block calculation. In turn, it is caused by the fact that the approximation formula for the viscosity contains pressure derivative $d p / d x$, which is very large near the kink of the profile. As a result, large values of viscosity are transported downwind causing the change of the form of the separation zone.

Therefore, a modified approximation of the turbulent viscosity is used, in which the contribution of $d p / d x$ is taken into account only where the geometry of the profile is sufficiently smooth. A reasonably good agreement is seen for both flow filed and skin friction in Figs 6 and 7 . The jump at $x / h \approx-2$ is explained by the profile kink.

\subsection{Flow over compression corner}

The final test for the ANDD scheme is the flow over compression corner $\alpha=$ $24^{\circ}$ with $M=1.5$ and $\operatorname{Re}=6 \cdot 10^{6}$ per meter. Fig. 8 shows the mesh of this case. This is another test which is meant to illustrate the efficiency of the method as applied to compressible flows with separation. The corner has the hypotenuse of $1.5 \mathrm{~m}$, the length of the plate before the corner is $5 \mathrm{~m}$, 


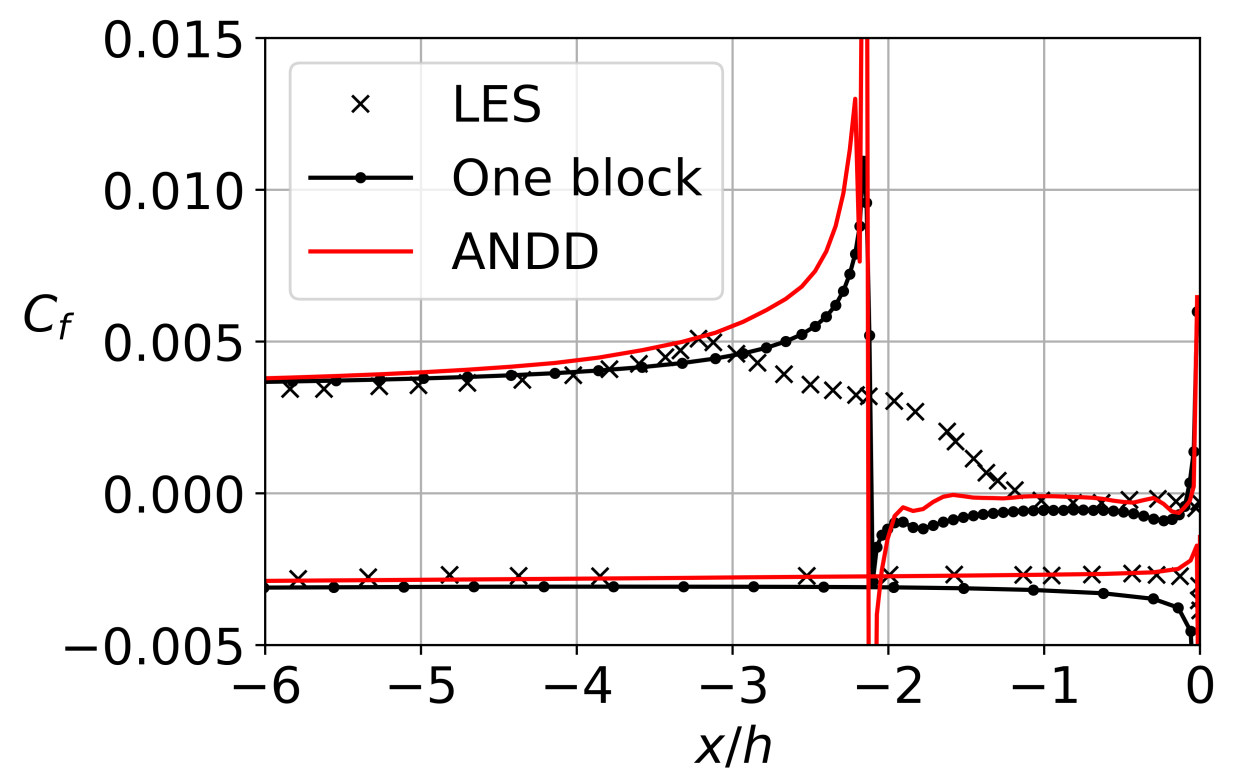

Figure 7. Skin friction coefficient along the surface of the profile with a sharp edge. Symbols correspond to the averaged LES data from [9]

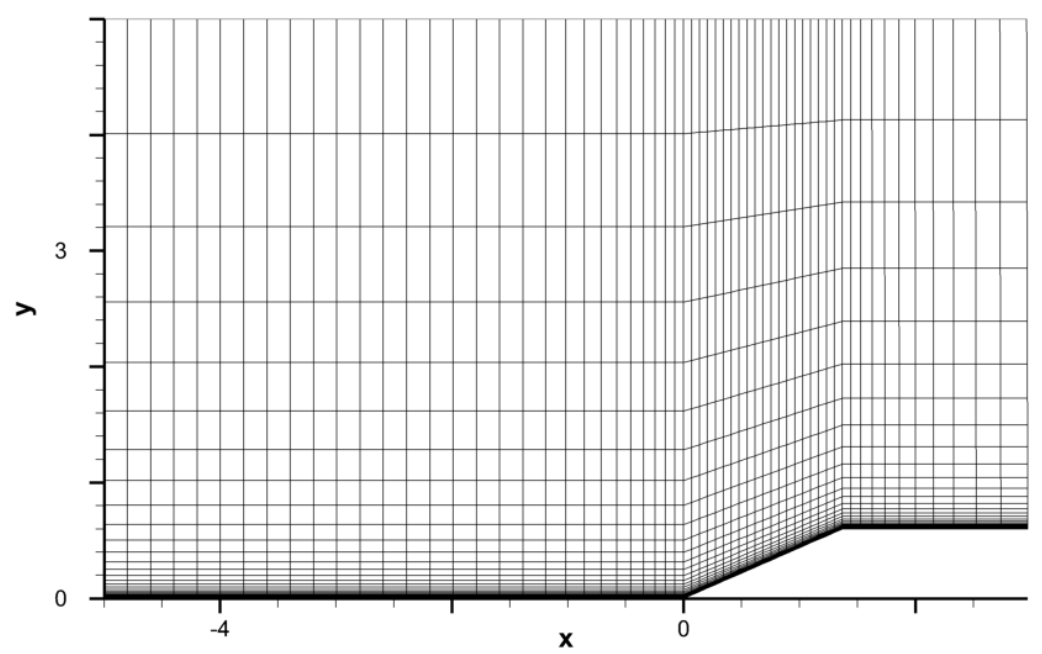

Figure 8. Flow over compression corner mesh

after it, $2 \mathrm{~m}$. The free-stream flow is parallel to the plates. In the one-block solution, the height of the first cell in the boundary layer is $10^{-6} \mathrm{~m}$. For the two-block run the distance from the interface boundary to the wall corresponds to $y^{*+}=10 \div 100$. As the turbulent variable, for the profile in the two-block 
calculation, SA solution (18) is used.

Fig. 9 shows skin friction coefficient on the corner surface computed by the one-block and ANDD methods. The first jump corresponds to the position of the shock wave $(x \approx-1.6)$ whereas the second one, to the passage from the corner to the plate surface $(x \approx 1.37)$. The start of the corner corresponds to $x \approx 0$. Separation position corresponds to $x \approx 0.76$ (see Fig. 10 ).

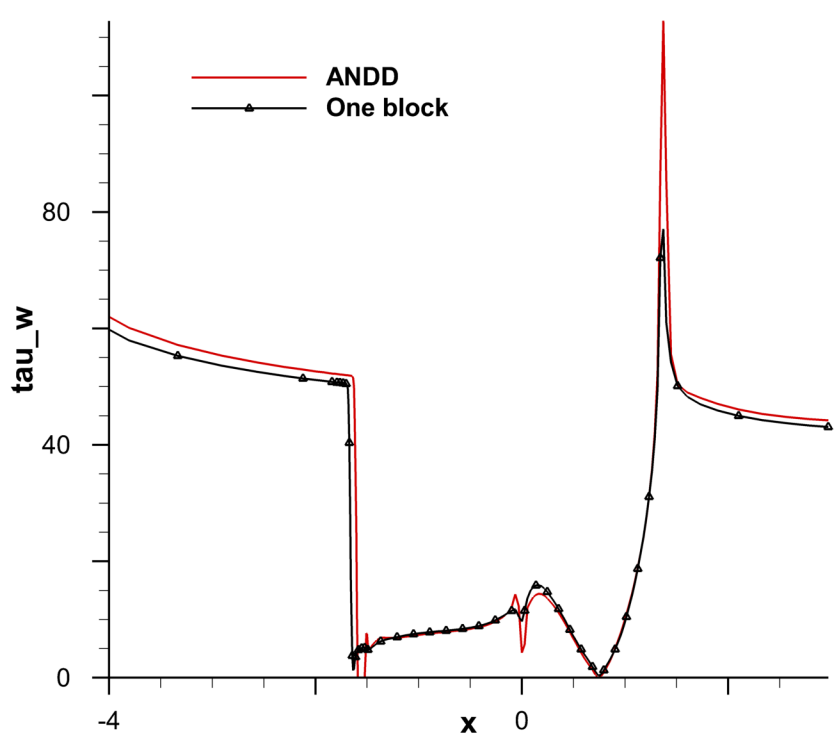

Figure 9. Skin friction on the surface of the compression corner from one-block and ANDD computations

It can be seen that the friction agrees well everywhere. Separation and shock front positions are computed with high accuracy as well. It should be noted that in this calculation $y^{+}$is almost everywhere below 100 . 


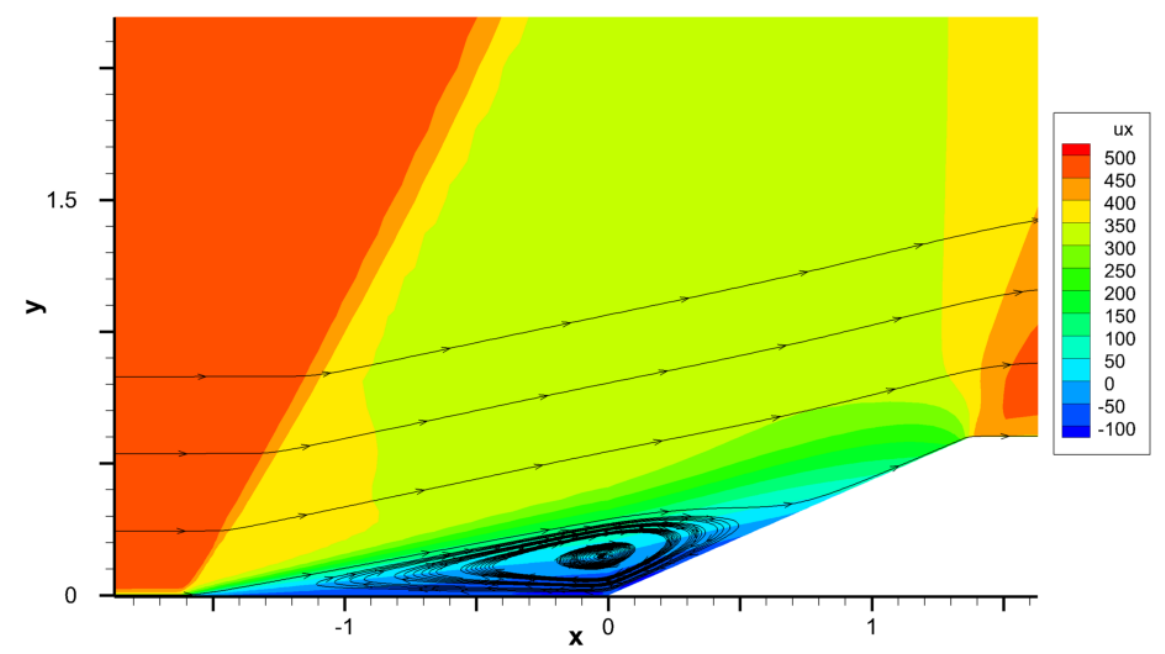

Figure 10. Velocity magnitude contour levels near the corner

\section{Conclusions}

In this work the method of the near-wall decomposition has been extended to the turbulent compressible flows with the closure by means of the SpalartAllmaras model. The proposed modification extends the capabilities of the method to supersonic flows. Test flows over blunted plate, half cylinder, compression corner and flow past a sharp edge demonstrate the possibility to compute with high accuracy flow fields and reconstruct density profile in the near-wall region. The density profiles shown in the paper for the flow over the blunted plate near the stagnation point demonstrate good agreement in the region where compressibility effects are important. Our study shows that skin friction for the considered geometries agrees with high accuracy between one-block calculation and two-block calculation with decomposition. A slight difference is found in the skin friction if the interface boundary is set at $y^{*+}>100$. The computational cost of the ANDD method is about three times lower as compared to the one-block calculation. Computations for half 
cylinder and compression corner demonstrate the capability of the method of the near-wall decomposition to predict with high accuracy the position of the separation point and the formation of the shock wave. It should be noted that the standard wall functions cannot fully resolve these effects.

The ENDD extends the capabilities of the NDD approach for improvement of the accuracy of calculations. Possible discrepancies between one-block and two-block calculations can be eliminated by changing from the approximate to the exact decomposition, carried in the framework of the same calculation and on the same mesh. ENDD can be efficiently implemented if ANDD is used to obtain an initial approximation. ENDD can be used for validation of solutions obtained with ANDD if needed. In addition, ENDD can supplement ANDD for the problems where the use of the latter approach is problematic such as flows with a laminar-turbulent transition and flows past complex geometries.

\section{Acknowledgement}

The work was supported by the Russian Science Foundation (Project N 1819-00098).

Some of the calculations were run on supercomputers of Joint Supercomputing Center of the Russian Academy of Sciences.

The authors are grateful to the referees for useful remarks which essentially improved the quality of the paper. 


\section{In memoriam}

This paper is dedicated to the memory of Dr. Douglas Nelson Woods (*January $11^{\text {th }} 1985$ - †September $11^{\text {th }} 2019$ ), promising young scientist and post-doctoral research fellow at Los Alamos National Laboratory. Our thoughts and wishes go to his wife Jessica, to his parents Susan and Tom, to his sister Rebecca and to his brother Chris, whom he left behind.

\section{References}

[1] Knopp, T. (2006). On grid-independence of RANS predictions for aerodynamic flows using model-consistent universal wall-functions. In Proceedings of ECCOMAS CFD.

[2] Aleksin, V. A., \& Utyuzhnikov, S. V. (2014). Implementation of near-wall boundary conditions for modeling boundary layers with free-stream turbulence. Applied Mathematical Modelling, 38(14), 3591-3606.

[3] Patankar, S. V., Spalding, D. B. (1967) Heat and mass transfer in boundary layers. London: Morgan-Grampian Press.

[4] Launder, B.E., Spalding, B.I. (1974) The numerical computation of turbulent flows. Computer Methods in Applied Mechanics and Engineering, 3, 269-289.

[5] Grotjans, H., \& Menter, F. R. (1998). Wall functions for industrial applications. Computational fluid dynamics, 98(1), 2.

[6] Kalitzin, G., Medic, G., Iaccarino, G., \& Durbin, P. (2005). Near-wall behavior of RANS turbulence models and implications for wall functions. Journal of Computational Physics, 204(1), 265-291. 
[7] Craft, T. J., Gerasimov, A. V., Iacovides, H., \& Launder, B. E. (2002). Progress in the generalization of wall-function treatments. International Journal of Heat and Fluid Flow, 23(2), 148-160.

[8] Craft, T. J., Gant, S. E., Iacovides, H., \& Launder, B. E. (2004). A new wall function strategy for complex turbulent flows. Numerical Heat Transfer, Part B: Fundamentals, 45(4), 301-318.

[9] Cabot, W., \& Moin, P. (2000). Approximate wall boundary conditions in the large-eddy simulation of high Reynolds number flow. Flow, Turbulence and Combustion, 63(1-4), 269-291.

[10] Duprat, C., Balarac, G., Métais, O., Congedo, P. M., \& Brugiere, O. (2011). A wall-layer model for large-eddy simulations of turbulent flows with/out pressure gradient. Physics of fluids, 23(1), 015101.

[11] Utyuzhnikov, S. V. (2005). Some new approaches to building and implementation of wall-functions for modeling of near-wall turbulent flows. Computers \& fluids, 34(7), 771-784.

[12] Utyuzhnikov, S.V. (2005). Generalized wall-functions and their application for simulation of turbulent flows, Int. J. Numerical Methods in Fluids, 47 (10-11): $1323-1328$.

[13] Utyuzhnikov S.V. (2006). The method of boundary condition transfer in application to modeling near-wall turbulent flows, Int. J. Computers $\&$ Fluids, 35 (10): 1193-1204.

[14] Utyuzhnikov S.V. (2008). Robin-type wall functions and their numerical implementation, J. Applied Numerical Mathematics, 58 (10): 1521-1533.

[15] Utyuzhnikov, S.V. (2012). Interface boundary conditions in near-wall turbulence modeling, Int. J. Computers \& Fluids, 68: 186-191. 
[16] Utyuzhnikov S.V. (2009). Domain decomposition for near-wall turbulent flows, Int. J. Computers $\&$ Fluids, 38 (9): 1710-1717.

[17] Utyuzhnikov, S.V. (2014). Towards development of unsteady near-wall interface boundary conditions for turbulence modelling. Comp. Phys. Communic., 185, $11,2879-2884$.

[18] Jones, A., Utyuzhnikov, S.V. (2015). Application of a near-wall domain decomposition method to turbulent flows with heat transfer. Computers $\mathcal{E}$ Fluids, 119, 87-100.

[19] Petrov, M. N., Tambova, A. A., Titarev, V. A., Utyuzhnikov, S. V., \& Chikitkin, A. V. (2018). FlowModellium software package for calculating high-speed flows of compressible fluid. Computational Mathematics and Mathematical Physics, 58(11), 1865-1886.

[20] Chikitkin, A., Petrov, M., Titarev, V., \& Utyuzhnikov, S. (2018). Parallel Versions of Implicit LU-SGS Method. Lobachevskii Journal of Mathematics, 39(4), 503-512.

[21] Petrov, M. N., Titarev, V. A., Utyuzhnikov, S. V., \& Chikitkin, A. V. (2017). A multithreaded OpenMP implementation of the LU-SGS method using the multilevel decomposition of the unstructured computational mesh. Computational Mathematics and Mathematical Physics, 57(11), 1856-1865.

[22] Jones, A., Utyuzhnikov, S.V. (2016). A near-wall domain decomposition approach in application to turbulent flow in a diffuser. Applied Mathematical Modelling, 40, (1): 329-342.

[23] Jones, A., Utyuzhnikov, S.V. (2017). Efficient computation of turbulent flow in ribbed passages using a non-overlapping near-wall domain decomposition method, Computer Physics Communications, 217: 1-10. 
[24] Utyuzhnikov, S., Smirnova, N. (2020). Unsteady interface boundary conditions for near-wall turbulence modeling, Computers and Mathematics with Applications, 79 (5): 1483-1502.

[25] L. Eça, Hoekstra M., Hay A., Pelletier D. (2007). A manufactured solution for a two-dimensional steady wall-bounded incompressible turbulent flow. International Journal of Computational Fluid Dynamics, 21(3-4), 175-188.

[26] Utyuzhnikov, S., Petrov, M., Chikitkin, A., Titarev, V. (2019). On extension of near-wall non-overlapping domain decomposition to essentially unsteady turbulent flows. Smart Innovation, Systems and Technologies. Proceedings of the Conference 50 Years of the Development of Grid-Characteristic Method. V. 133. P. 199 - 209. Springer, Cham.

[27] Yoon, S., \& Jameson, A. (1988). Lower-upper symmetric-Gauss-Seidel method for the Euler and Navier-Stokes equations. AIAA journal, 26(9), 1025-1026.

[28] Menshov, I. (1995). An Implicit Advection Upwind Splitting Scheme for Hypersonic Air Flows in Thermomechanical Nonequilibrium. In 6th Int. Symp. on CFD, 1995 (Vol. 2, pp. 815-820).

[29] Chikitkin, A.V., Utyuzhnikov, S.V., Petrov, M.N., Titarev, V.A. (2020). Nonoverlapping Domain Decomposition for Modeling Essentially Unsteady Nearwall Turbulent Flows, Computers and Fluids, 202:104506

[30] Lions, P.L. (1990). On the Schwarz alternating method III: a variant for nonoverlapping subdomains, in Third International Symposium on Domain Decomposition Methods for Partial Differential Equations, T.F. Chan, R. Glowinski, J. Periaux, and O.B. Widlund, eds., SIAM, Philadelphia, PA, pp. $202-223$.

[31] Deng, Q. (1997) A analysis for a nonoverlapping domain decompositon iterative procedure . SIAM J. Sci. Comp. 18: 1517-1525. 
[32] Lui, S. H. (2001). On accelerated convergence of nonoverlapping Schwarz methods. Journal of computational and applied mathematics 130.1-2 : 309-321.

[33] Utyuzhnikov, S. (2019). Chen, W. Exact non-overlapping domain decomposition for near-wall turbulence modeling. Computers \& Fluids 181 : 283-291.

[34] Duprat, C., Balarac, G., Metais, O., Congedo, P. M., \& Brugiere, O. (2011). A wall-layer model for large-eddy simulations of turbulent flows with/out pressure gradient. Physics of Fluids, 23(1), 015101.

[35] Allmaras, S. R., Forrester T. J. (2012). Modifications and clarifications for the implementation of the Spalart-Allmaras turbulence model. Seventh international conference on computational fluid dynamics (ICCFD7).

[36] Blake, W. K. (1975). A statistical description of pressure and velocity fields at the trailing edges of a flat strut (No. DTNSRDC-4241). David W Taylor naval ship research and development center Bethesda md. 